\author{
J.-M. Lemoyne de Forges \\ Professeur émérite de l'Université, Panthéon-Assas (Paris II)
}

\title{
LE CONTRÔLE DE L'ADMINISTRATION PAR LES INSPECTIONS MINISTÉRIELLES ET INTERMINISTÉRIELLES
}

\author{
UDK: $354.11 / .85$ (44) \\ Pregledno znanstveno priopćenje \\ Primljeno: 15: IX. 2015.
}

\begin{abstract}
D'origine fort ancienne, les corps d'inspection se sont multipliés au $\mathrm{XIX}^{\circ}$ et $\mathrm{XX}^{\circ}$ siècle et les missions de la plupart d'entre eux ont sensiblement évolué depuis une trentaine d'années. Jadis chargés de veiller d'une part à la bonne exécution des instructions gouvernementales ou ministérielles et d'autre part à la régularité juridique, budgétaire et comptable de l'activité de l'administration, ils sont aujourd'hui souvent chargés en outre de missions d'évaluation de l'efficacité des politiques publiques, voire de propositions de réformes administratives.

Dotés d'un statut qui combine leur indépendance fonctionnelle et leur rattachement direct à un ou plusieurs ministres, les membres de tous ces corps d'inspection ont des méthodes de travail particulières, adaptées à la nature de leurs différentes fonctions.

Les inspections plus importantes ont une vocation interministérielle (inspection générale des finances, inspection générale de l'administration, inspection générale des affaires sociales) ; les plus nombreuses sont propres à chacun des grands ministères (défense, justice, affaires étrangères, éducation nationale, environnement...).
\end{abstract}

\section{Mots - clés: Les corps d'inspection, active de l'administratif, France}

On fait habituellement remonter l'origine des corps d'inspection à l'institution, par Charlemagne, des « missi dominici », envoyés par le roi pour veiller à l'application uniforme de certaines de ses lois (les « capitulaires ») dans l'ensemble du royaume. Par la suite, et jusqu'au début du XIX ${ }^{\circ}$ siècle, d'autres types d'inspections ont été créées, tantôt dans le même but d'assurer l'exécution des décisions du pouvoir central ( représentants en mission » de la Convention en 1793), tantôt pour vérifier les comptes des administrations (Inspection générale des finances en 1814), tantôt pour veiller à la qualité des services publics («Inspecteurs généraux des études » de 1802).

De ces origines, on a conservé l'idée que les missions des corps d'inspection français contemporains, devenus très nombreux, peuvent être très variées. Certes, l'objectif de contrôle de la régularité juridique et financière du fonctionnement de l'administration reste très important; toutefois, surtout depuis une trentaine d'années, presque tous les corps d'inspection ont vu leur mission élargie au contrôle de la qualité, de l'efficacité et de l'efficience des services de l'État, voire au conseil des membres du gouvernement en matière de réforme administrative. 
Avant d'entrer plus avant dans l'analyse du rôle et du statut des corps d'inspection, il faut souligner que, conformément d'ailleurs à ses obligations communautaires ${ }^{1}$, chaque ministère français doit, depuis un décret du 28 juin $2011^{2}$ , être doté d'un dispositif d'audit interne, inspiré de ce qui se pratique dans les grandes entreprises privées, visant à maîtriser les risques liés à la réalisation des objectifs du ministère et notamment à vérifier que les dispositifs de contrôle interne, y compris l'activité des corps d'inspection, sont efficaces. Les chefs des corps d'inspection ministériels participent d'ailleurs systématiquement au « comité d'audit » du ministère dont ils relèvent.

\section{QUELS SONT LES PRINCIPAUX CORPS D'INSPECTION ?}

Trois grands corps d'inspection ont une compétence interministérielle ; il arrive même souvent que, selon la nature et l'importance du sujet traité, des missions d'inspection soient composées d'inspecteurs provenant de deux de ces corps, voire des trois, ou encore d'inspecteurs provenant de l'un ou l'autre de ces trois grands corps d'inspection et de membres des inspections ministérielles, en principe plus spécialisés.

Le plus ancien (1814), le plus prestigieux, le plus recherché (en termes de carrière administrative) et le plus connu est le corps de l'Inspection générale des finances (IGF), placé sous l'autorité directe du ou des ministres chargés de l'économie et des finances et dont le rôle est défini en termes très larges, englobant le contrôle, l'audit, l'étude, le conseil et l'évaluation en matière administrative, économique et financière.

Le chef du service de l'Inspection générale des finances gère le corps et répartit les missions entre ses membres, missions qui répondent souvent à des commandes du Premier ministre ou d'autres ministres, en particulier en matière d'études et d'évaluation. Mais une large part de l'activité de l'IGF correspond à des programmes de vérification, de contrôle ou d'audit décidés par le corps luimême ou par le comité d'audit interne des ministères économiques et financiers.

Toutefois, le caractère interministériel de l'IGF résulte du fait que tous les services à compétence financière de l'État, services centraux et services déconcentrés de tous les ministères, ordonnateurs des recettes et des dépenses aussi bien que comptables publics, relèvent de son contrôle. Traditionnellement, pour assurer ces missions de contrôle, l'IGF dispose de « tous pouvoirs d'investigation sur pièces et sur place $»^{3}$, héritage de l'époque où tous les jeunes inspecteurs commençaient leur carrière en vérifiant les recettes et les dépenses de l'État en se rendant sur place, sur l'ensemble du territoire national, pour contrôler les services déconcentrés du ministère des finances.

${ }^{1}$ V. Directive Conseil no 2011/85/UE, art. 3 : « Les Etats membres disposent de systèmes de comptabilité publique (...) soumis à un contrôle interne et à un audit indépendant ».

${ }^{2}$ Décret $n^{\circ} 2011-775$ relatif à l'audit interne dans l'administration.

${ }^{3}$ Décret n$^{\circ}$ 55-733 du 26 mai 1955 relatif au contrôle économique et financier de l’État, art. 8. 
Remontant à 1848 mais ayant acquis son appellation actuelle en 1948, l'Inspection générale de l'administration (IGA) est placé sous l'autorité du ministre de l'Intérieur. Ses missions de base sont définies à peu près dans les mêmes termes que celles de l'IGF ( « mission générale de contrôle, d'audit, d'étude, de conseil et d'évaluation à l'égard des services centraux et déconcentrés qui relèvent du ministre de l'Intérieur $»^{4}$ ), mais elles s'étendent à tous les personnels, services, institutions et organismes sur lesquels les préfets exercent leur contrôle, même lorsqu'ils sont soumis aux vérifications d'un autre corps d'inspection ou de contrôle spécialisé ; l'IGA peut encore assurer des missions d'évaluation des politiques publiques, de formation, de coopération internationale ; enfin, des collectivités territoriales, des fondations, des associations, des Etats étrangers ou des organisations internationales peuvent faire appel à ses compétences.

Issue de la fusion, en 1967, de plusieurs corps d'inspection spécialisés (dont certains d'origine fort ancienne), l'Inspection générale des affaires sociales (IGAS) est placée sous l'autorité des ministres chargés des affaires sociales (santé, sécurité sociale, action sociale, famille, travail, emploi, formation professionnelle) mais peut aussi recevoir des missions directement du Premier ministre. Ses fonctions sont, dans son très vaste domaine de compétence, définies largement (missions d'inspection, de contrôle et d'audit, missions d'enquête et d'évaluation, missions de conseil et d'appui ${ }^{5}$ ).

Quant aux inspections générales ministérielles, par principe plus spécialisées, elles sont d'autant plus nombreuses que certains ministères en comptent plusieurs. Ainsi, en matière d'enseignement et de recherche, à côté de l'Inspection générale de l'éducation nationale, chargée de la pédagogie, on trouve l'Inspection générale de l'administration de l'éducation nationale et de la recherche, qui contrôle le bon fonctionnement de l'ensemble des institutions d'enseignement et de recherche, ou encore l'Inspection générale des bibliothèques, évidemment plus spécialisée. De même, au ministère de la Défense, se rencontrent l'Inspection générale des armées, mais aussi le Contrôle général des armées (dont les fonctions, très larges, s'apparentent à celles des grandes inspections interministérielle) et l'Inspection générale du service de santé des armées. Pour les autres ministères, on se bornera à quelques exemples : l'Inspection générale des affaires étrangères (qui contrôle notamment le bon fonctionnement des ambassades), l'Inspection générale des affaires culturelles, l'Inspection générale de l'agriculture, l'Inspection générale des affaires maritimes, l'Inspection générale de la police nationale, ou encore l'Inspection générale de la jeunesse et des sports.

$\mathrm{Au}$ total, il existe 27 « inspections générales » réunissant environ 2.000 fonctionnaires, auxquels il faut ajouter les nombreuses inspections placées à un niveau hiérarchique moins élevé, parmi lesquelles on peut citer à titre d'exemples

${ }^{4}$ Décret $n^{\circ}$ 81-241 du 12 mars 1981 portant statut de l'inspection générale de l'administration au ministère de l'intérieur, art. 1er .

${ }^{5}$ V. Décret $n^{\circ}$ 2011-931 du 1er août 2011 portant statut particulier du corps de l'inspection générale des affaires sociales. 
l'Inspection du travail (2.000 agents qui surveillent notamment l'application du droit du travail dans les entreprises), les inspecteurs des finances publiques (qui veillent en particulier à la bonne application des règles fiscales), les inspecteurs de santé publique ou les inspecteurs d'académie (surtout chargés contrôler les aspects pédagogiques de l'activité des enseignants). Sachant que les fonctionnaires de l'État sont au nombre d'environ 2 millions (y compris les enseignants, qui représentent près de la moitié de ce total) et que la fonction publique hospitalière compte près d'un million de fonctionnaires ${ }^{6}$, l'ensemble des corps d'inspection de l'État de tous niveaux ayant compétence sur les services de l'État et sur les services publics de santé représente entre $0,5 \%$ et $1 \%$ des fonctionnaires de l'État, dont les 27 inspections générales ministérielles et interministérielles ne représentent qu'environ $0,01 \%$ des fonctionnaires de l'Etat $^{7}$

\section{QUEL EST LE STATUT DES CORPS D'INSPECTION ?}

La qualité et l'efficacité des inspections de l'État dépendent principalement de deux facteurs : la qualité technique de leurs membres et leur indépendance. Ces deux garanties sont bien connues des entreprises privées qui, outre leurs contrôles internes, font appel à des cabinets d'étude, d'audit et de conseil extérieurs. Dès lors que, par hypothèse, les inspections sont internes à l'administration et que leurs missions ont pour principal objet la régularité, la qualité, l'efficacité et l'efficience de l'administration elle-même, ces deux garanties doivent faire l'objet de précautions institutionnelles et juridiques particulières.

Pour garantir la qualité du recrutement, le modèle français obéit aux principes suivants.

En ce qui concerne les trois inspections générales interministérielles (IGF, IGA et IGAS) le recrutement est assuré principalement par l'Ecole nationale d'administration. En majorité, ces inspecteurs commencent donc leur carrière très jeunes. Toutefois, l'attrait de ces corps vient aussi du fait que, au cours de leur carrière, ils auront de nombreuses occasions d'exercer des fonctions d'administration active à des niveaux de responsabilité élevée (notamment grâce à la procédure de " détachement »), ce qui doit enrichir leurs compétences quand ils reviennent dans leur corps d'inspection. Toutefois, pour diversifier le recrutement, entre un quart et un tiers des membres, selon le corps, est recruté parmi des fonctionnaires plus âgés, plus expérimentés et de formation différente. En particulier, selon ses statuts, l'IGAS doit toujours compter au moins $10 \%$ de docteurs en médecine ou en pharmacie et $4 \%$ d'anciens inspecteurs du travail. Ces recrutements sont assurés par un comité de sélection propre au corps qui apprécie

\footnotetext{
${ }^{6}$ Les fonctionnaires des collectivités locales sont environ 1,5 millions. Tous ces chiffres (approximatifs) ne concernent que les « fonctionnaires » au sens strict; on peut y ajouter les autres agents «non titulaires » de l'administration (environ 300.000 pour l'État, 110.000 pour les hôpitaux et 250.000 pour les collectivités locales).

${ }^{7}$ Certaines grandes villes (en particulier Paris) et certaines régions ont par ailleurs créé leurs propres corps d'inspection.
} 
les qualités des candidats et soumet ses propositions aux ministres compétents pour les nommer officiellement. Selon un usage bien établi, les ministres suivent les propositions de ces comités de sélection; de plus, en cas d'erreur «manifeste » d'appréciation sur ces qualités, le Conseil d'État peut être conduit à annuler la nomination.

Pour les inspections générales ministérielles, le recrutement se fait pratiquement seulement parmi des fonctionnaires (voire, dans quelques cas, des personnalités extérieures à l'administration) très expérimentés, donc plutôt comme un couronnement de carrière. Le statut de chaque corps précise quelles sont les catégories de personnes autorisés à postuler (parfois avec un nombre limité d'emplois pour chaque catégorie), voire les expériences professionnelles exigées des candidats. Ici encore, l'usage est que les ministres compétents suivent les propositions des comités de sélection institués au sein de chaque corps, le cas échéant sous le contrôle du Conseil d'État. On comprend bien que toutes ces précautions juridiques sont destinées non seulement à garantir la qualité du travail du corps d'inspection mais aussi à éviter les risques de favoritisme politique, syndical ou amical ; c'est d'autant plus important que les rémunérations des membres de ces corps d'inspection générale sont parmi les plus élevées de la fonction publique. Cela dit, la presse se fait cependant périodiquement l'écho de quelques cas où le pouvoir exécutif parvient à faire nommer des personnalités qui, tout en remplissant les conditions d'expérience fixées par le statut du corps, ont en outre la qualité d'amis politiques...

Ces corps d'inspection doivent évidemment être indépendants, tant du pouvoir politique que de tout autre forme de pression imaginable. L'indépendance est d'ailleurs aussi une garantie de la qualité de leur travail. Dans la tradition française, cette indépendance est assurée par une véritable autogestion de chaque corps. On vient de le voir pour ce qui est du recrutement, mais cela concerne aussi le programme et les méthodes de leur travail et dans une large mesure la carrière administrative de ses membres.

Certes, le programme de travail des corps d'inspection générale répond souvent à des commandes des ministres ou du Premier ministre. Mais une grande partie de ce programme est décidé, en principe chaque année, par le chef de chaque corps sur la base d'une concertation entre ses membres. Et les corps d'inspection générale recourent largement à cette faculté de décider eux-mêmes sur quels sujets porteront leurs investigations. Toutefois, leurs rapports sont destinés aux seuls membres du gouvernement qui décideront de leur publication éventuelle ${ }^{8}$ et, le cas échéant, de la suite qu'il convient de leur donner, notamment en termes de réformes administratives voire, dans certains cas, de poursuites disciplinaires contre des fonctionnaires qui auraient manqué à leur obligations.

Le statut particulier de chaque corps d'inspection générale réglemente le

${ }^{8}$ Etant cependant précisé que toutes les inspections générales publient chaque année un rapport d'activité qui rend compte des travaux réalisés au cours de l'année et des enseignements tirés des observations effectuées lors des missions du corps. 
déroulement de la carrière administrative de ses membres d'une manière très précise, d'autant plus différente pour chaque corps que leurs effectifs ne dépasse guère 100 personnes $^{9}$, la plupart des inspections générales ministérielles ne comptant que quelques dizaines de membres. Pour simplifier, on notera seulement que, pour éviter le favoritisme, d'une part l'avancement au sein du corps fait une large part à l'ancienneté et d'autre part chaque corps est doté d'une «commission administrative paritaire » qui fait ses propositions aux ministres compétents pour les changements de grade (inspecteur de 1ère classe, inspecteur général) ${ }^{10}$. C'est l'ensemble petits effectifs, rémunérations élevées, comités de sélection, avancement à l'ancienneté et poids des commissions administratives paritaires qui assure l'indépendance (et le prestige) des inspections générales ministérielles et interministérielles.

\section{QUELLES SONT LES MÉTHODES DE TRAVAIL DES INSPECTIONS GÉNÉRALES ?}

La diversité des inspections générales et de leurs attributions interdit de donner une présentation détaillée de ces méthodes de travail : il est évident qu'un contrôle de régularité financière ne peut être réalisé de la même manière qu'une mission d'évaluation d'une politique publique.

On peut cependant donner quelques repères pratiques.

En premier lieu, chaque corps d'inspection élabore lui-même un ou plusieurs « guides méthodologiques » ou des « référentiels » adaptés aux différents types de mission confiés au corps. Ceci est destiné d'une part à garantir l'homogénéité des méthodes de travail du corps, et d'autre part à informer les services, institutions et fonctionnaires des conditions dans lesquelles sera conduite la mission de contrôle, d'inspection, d'audit ou d'évaluation dont ils vont être l'objet.

En deuxième lieu, chaque mission est toujours collégiale. On a déjà signalé qu'il arrivait souvent qu'une même mission soit confiée à des membres de deux ou trois corps d'inspection. Mais même lorsqu'il s'agit d'une mission confiée à un seul corps d'inspection, elle est toujours assurée par au moins deux inspecteurs désignés par le chef du corps, dont les compétences sont aussi complémentaires que possible; en outre ces inspecteurs sont souvent assistés de quelques fonctionnaires de niveau hiérarchique moins élevé.

En troisième lieu, les missions d'inspection font une large part au recueil d'informations, par auditions et par écrit, auprès de toutes les personnes susceptibles de fournir des informations pertinentes aux inspecteurs. Selon la

9 Non compris, cependant, les membres de ces corps qui sont détachés temporairement pour exercer des fonctions d'administration active, voire appelés à travailler dans des entreprises publiques ou même privées.

${ }^{10}$ Tous les corps de fonctionnaires sont dotés d'une telle « commission administrative paritaire » (CAP) mais la tradition leur donne plus ou moins d'influence selon les corps. Ainsi, les avis des CAP sont presque toujours suivis dans les corps d'inspection générale et dans les corps enseignants. 
nature de la mission, le nombre et la qualité de ces interlocuteurs sont évidemment très variables.

En quatrième lieu, les inspecteurs ont toujours des pouvoirs étendus pour pénétrer dans tous les locaux administratifs, se faire communiquer tout document dont ils estiment avoir besoin et auditionner toute personne de leur choix. Du reste, le fait de faire obstacle au travail d'enquête d'une équipe d'inspecteurs est dans un grand nombre de cas un délit passible de sanctions pénales ${ }^{11}$.

En cinquième lieu, dans tous les cas où la mission consiste à analyser - et donc forcément à critiquer - le fonctionnement d'une institution ou d'un service administratif, la procédure suivie est toujours contradictoire : les inspecteurs présentent au service contrôlé un pré-rapport sur lequel ce service est invité à présenter ses observations avant que les inspecteurs n'établissent leur rapport définitif dans lequel soit ils tiennent compte de la «défense » du service, soit ils expliquent pourquoi ses observations doivent être écartées.

Enfin, l'efficacité des missions d'inspection est assurée par quelques dispositifs complémentaires. Ainsi, si la plupart des missions sont préparées et annoncées à l'avance, certaines opérations de contrôle sur pièces et sur place sont réalisées à l'improviste, sans que le service contrôlé en soit prévenu avant l'arrivée des inspecteurs ${ }^{12}$. Ainsi encore, les résultats des audits, contrôles et autres formes d'inspection sont toujours communiqués aux responsables des services contrôlés afin de les inciter à tenir compte des critiques et des propositions d'amélioration présentées par les inspecteurs. Enfin, plusieurs corps d'inspection ont constitué une « cellule de suivi » chargée d'examiner périodiquement les suites données à ces critiques et à ces propositions.

\section{CONCLUSION}

L'extension des missions des inspections générales ministérielles et interministérielles, notamment des missions d'évaluation des politiques publiques, d'audit et de conseil, ne permet pas d'entrer dans le détail du fonctionnement réel de ces inspections.

On conclura donc simplement par deux remarques complémentaires.

La première est que, comme la Cour des comptes, ces corps d'inspection travaillent tantôt sur des sujets très précis, étroits et techniques, tantôt sur des politiques publiques d'une certaine envergure. On en donnera en annexe un exemple (V. P.J.).

La seconde est que si, comme d'ailleurs pour la Cour des comptes, on s'interroge souvent sur l'utilité réelle des innombrables rapports élaborés par les corps

${ }^{11}$ V. not. art. 43 de la loi n96-314 du 12 avril 1996 portant diverses dispositions d'ordre économique et financier pour l'IGF, l'IGA, l'IGAS et quelques inspections générales ministérielles.

12 Par exemple, c'est le mode de travail normal des inspections réalisées auprès des unités militaires par le corps du « Contrôle général des armées ». 
d'inspection générale, force est de constater que nombre de mesures d'amélioration du fonctionnement de l'administration trouvent leur origine dans ces rapports, que les fonctionnaires, en tout cas les chefs de service, craignent toujours quelque peu l'intervention d'une équipe d'inspecteurs, que personne n'envisage de supprimer le moindre corps d'inspection, ni même d'alléger leurs missions, et enfin que des administration étrangères ou des institutions internationales font volontiers appel aux inspections générales françaises ${ }^{13}$.

\author{
13 Annexes : \\ page 9 : extraits du Rapport d'activité de l'IGA 2014 \\ page 10 : schéma d`un rapport d`inspection contradictoire

\section{INSPECTION GÉNÉRALE DE L〉ADMINISTRATION RAPPORT D>ACTIVITÉ 2014 (extraits)}

\title{
PRÉSENTATION DE L〉I.G.A.
}

65 membres en activité à l'I.G.A. (dont les $2 / 3$ issus de l'ENA)

125 rapports (dont 25 rendus publics)

1157 journées de déplacements hors Ile-de-France

12 missions permanentes (p. ex. contrôle de l'utilisation des fonds structurels e u r o p é e n s, évaluation des politiques de prévention de la délinquance, évaluation des services départementaux d'incendie et de secours...)

6 groupes référents (réforme de l'État, fonction publique, collectivités locales, sécurité civile, libertés publiques, sécurité intérieure)

\section{EXEMPLES DE RAPPORTS}

- Moderniser l'organisation des élections l'État

- Enseignements à tirer de la création de la Métropole de Lyon pour les missions et l'organisation de

- L'encadrement supérieur et dirigeant de l'État

- La qualité des procédures judiciaires confrontées à la délinquance de masse

- Les nouveaux usages de la route

- Evaluation des conditions d'accueil des ressortissants étrangers par les préfectures et les souspréfectures

-Traitement des demandes de titres de séjour des étudiants étrangers

- Evaluation des « zones de revitalisation rurale»

- L'accès aux livres dans les départements d'outre-mer

- Audit du contrôle interne des marchés d'assistance et de réalisation en matière informatique

- Evaluation de la politique de sécurité routière

- L'enregistrement des plaintes dans le ressort de la Préfecture de Police de Paris.

Pré-rapport/ Observations du service// Rapport définitif des inspecteurs 


\section{KONTROLA UPRAVE PUTEM MINISTARSKIH I MEĐUMINISTARSKIH INSPEKCIJA}

Iako su inspekcijska tijela vrlo stara, njihov broj povećao se u 19. i 20. stoljeću, a zadaće većine njih značajno su se promijenile u posljednjih tridesetak godina. Nekoć su imala zadatak brinuti o dobrom izvršenju vladinih ili ministarskih uputa s jedne strane, a s druge strane o pravnoj, proračunskoj i računovodstvenoj regularnosti djelovanja uprave. Danas su često odgovorna za procjenu učinkovitosti javnih politika i za prijedloge upravnih reformi.

Budući da imaju status koji kombinira njihovu funkcionalnu neovisnost i njihovu izravnu povezanost s jednim ili više ministara, članovi svih inspekcijskih tijela imaju posebne metode rada koje su prilagođene prirodi njihovih različitih dužnosti.

Najvažnije inspekcije djeluju na međuresornoj razini (glavni inspektorat za financije, glavni inspektorat za upravu, glavni inspektorat za socijalna pitanja), a najbrojnije pripadaju svakom od velikih ministarstava (obrana, pravosuđe, vanjski poslovi, nacionalno obrazovanje, okoliš...).

Ključne riječi: Inspekcijska tijela, djelatnost uprave, Francuska 